\title{
A Novel Approach for Detection of Microaneurysms in Diabetic Retinopathy Disease from Retinal Fundus Images
}

\author{
Nasr Y. Gharaibeh ${ }^{1}$ \\ ${ }^{1}$ Al-Balqa Applied University, Jordan \\ Correspondence: Nasr Y. Gharaibeh, Al-Huson University College/Al-Balqa Applied University, P.O. Box 50, \\ Al-Huson 21510, Jordan. Mobile: 962-775-612-971. E-mail: nsrghr@gmail.com
}

Received: October 21, 2015

Accepted: December 8, 2015

Online Published: December 25, 2016

doi:10.5539/cis.v10n1p1

URL: http://dx.doi.org/10.5539/cis.v10n1p1

\begin{abstract}
Diabetic Retinopathy (DR) is a leading cause of blindness in human beings aged between 20 to 74 years. It has a great influence on the patient and society because it normally influences humans in their most gainful years. Early detection in DR is very difficult which is not detected by human beings. Many algorithms and techniques were established to detect DR. These techniques faced the problems such as increasing sensitivity, specificity and accuracy. To overcome those problems we have to introduce an effective image processing algorithms for increasing performances and also easily identify the DR diseases. One of the most challenging tasks in screening is automatic detection of Microaneurysms (MAs). This paper presents a new approach to detect MAs. Our proposed work consists of preprocessing, blood vessel segmentation (FPCM), fovea localization, fovea elimination, feature extraction and classification (Neuro-Fuzzy). Neuro-Fuzzy is a combined version of neural networks and fuzzy logical models. Experiments are conducted using MATLAB simulation tool. Using MESSIDOR database for our experiments which provides efficient and effective results in sensitivity, specificity, correct classification and detection rate (accuracy) and precision.
\end{abstract}

Keywords: blood vessels, MESSIDOR database, fovea, microanueurysm

\section{Introduction}

Diabetic eye disease contains group of eye conditions that affects people with diabetes. These conditions include diseases such as diabetic retinopathy, diabetic macular edema, cataract and glaucoma these are very potential to cause severe vision loss problem and blindness problem in working age adults. Changes in blood vessels lead to the problem of diabetic retinopathy since it affects the lining of back of the eye. This will called as retina. DR has a significant impact on the world health organization systems. The number of people with DR will grow from 126.6 million in 2010 to 191.0 million by 2030. In general, DR is a silent disease (Rajan, 2015), (Kanika Verma, Prakash Deep \& Ramakrishnan, 2011) because this is identified by the patient when the level changes in the retina. Some common symptoms of diabetic retinopathy are given below:

- Blurred vision

- Fluctuating vision

- Impaired color vision

- Vision loss

- Floaters vision

- Flashers vision (dark spots)

- Vision in dark and empty areas

The effect of diabetic retinopathy on vision is varies widely, depending on the stage of the disease. Generally DR has two stages such as Proliferative Diabetic Retinopathy (PDR) and Non-Proliferative Diabetic Retinopathy (NPDR). PDR has the components of neovascularisation and vitreous fluid hemorrhage since new blood vessels grow on the surface of the retina and it can bleed. But in NPDR has no symptoms which are detect only by retinal photography. Diabetic NPDR stages are classified into three: Mild, moderate and severe (Latare \& Patil, 2015): 
- Mild NPDR: This kind of NPDR is also called as Micro aneurysms, which is small swellings in small blood vessels in the retina.

- Moderate NPDR: This is mainly progress based on blood vessels which nourished by the retina.

- Severe NPDR: This is due to large amount of blood vessels blocked and arrest blood supply into many retinal areas. This could cause lack of oxygen. Problem of these should raise both hemorrhages (Dot and Blot), bleeding in the veins and also intra retinal micro vascular abnormalities.

- PDR: This is unchecked progression PDR since it difficult to detect by Ophthalmologist (Walvekar \& Salunke, 2015). It can lead to transactional retinal attachment. The signal sent by the retina for nutrition stimulation of the growth of new blood vessels.

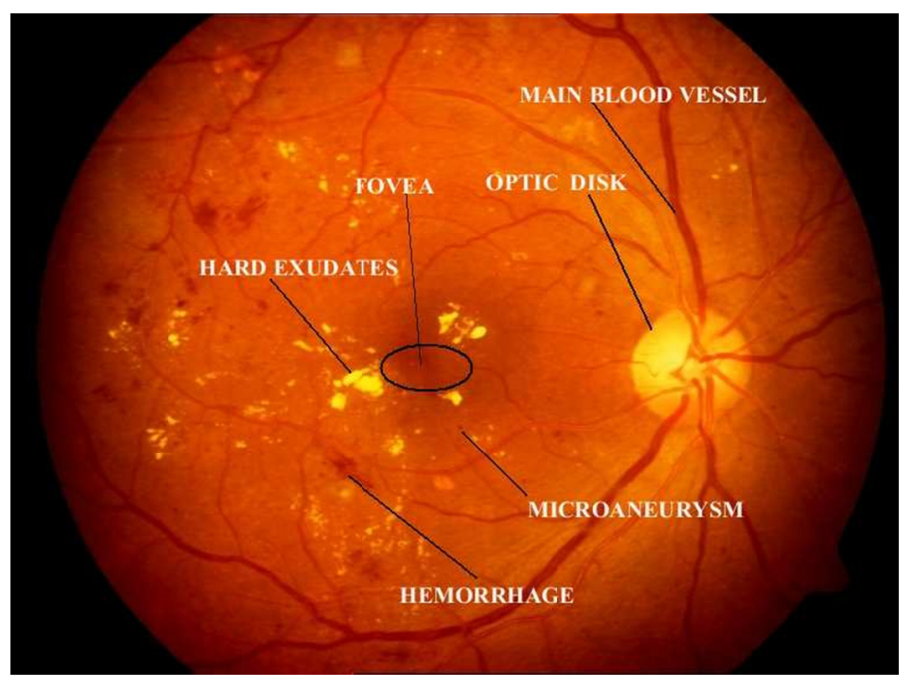

Figure 1. Illustration of Various Features on a Retinopathy Image

Figure 1 illustrates the various features on a retinal image. DR features/signs are the following: Microaneurysms, Hemorrhage, Hard exudates.

Microaneurysms: Red spots appear on the retinal layers due to pericyte loss and this is very small.

Hemorrhages: This is similar to microaneurysm this is small in size that will appear in deeper layer of the retina. Hemorrhages split into two types: dot hemorrhage and blot hemorrhage. Dot hemorrhage is differing from geometrical features and blot is same as in color, contrast and geometry.

Hard exudates: Exudates are bright region. It is similar to optic disc as in color which is placed nearby macula. Yellow spots appear on the retina.

Many techniques that detects/classifies the diabetic retinopathy they are soft computing techniques and supervised learning algorithms. Soft computing approaches are used for automatic detection and classification (Janakiraman \& Gowri, 2014). Aim of soft computing is to exploit the tolerance for imprecision and uncertainty to achieve tractability and low cost. Soft computing constitutes fuzzy logic concerned with imprecision and approximate reasoning, neural networks for learning and curve fitting, evolutionary algorithms achieves robustness and probabilistic graph models belief propagation and uncertainty figure. 2 shows the soft computing approach process. 


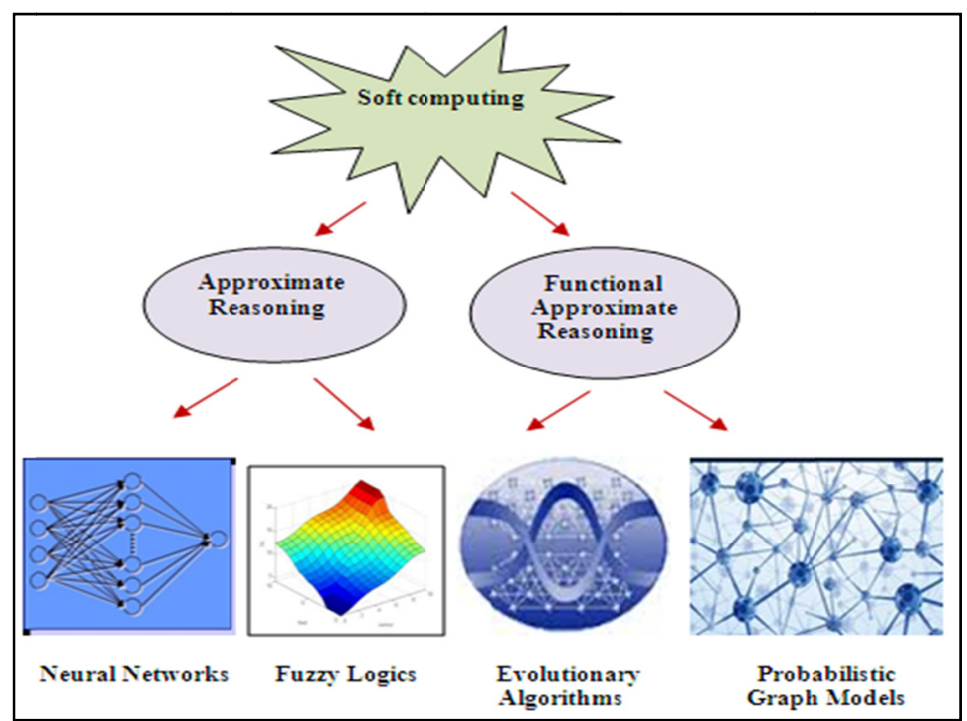

Figure 2. Soft Computing Approach

Contribution of our proposed work as follows:

- Retinal blood vessel segmentation based on Fuzzy Possibilistic C-Means (FPCM).

- Fovea localized by sliding window technique.

- Fovea eliminated by Morphological Operations.

- Feature extraction to be done by the Grey Level Co-occurrence Matrix and Logical Binary Patterns (GLCM \& LBP)

- Classification of diabetic retinopathy is Microaneurysms and Non-Microaneurysms which can be done with the help of Neuro-Fuzzy system.

The rest of this paper is organized as follows: Section 2 discussed related work, section 3 describes proposed work. Section 3 presents the experimental results and analysis and concluding remarks following in section 5 .

\section{Related Work}

Retinal images are used for detecting/classifying retinal diseases in an earlier manner. In (Ganesh \& Basha, 2015), (Maheswari \& Punnolil, 2014) clearly explained about diabetic retinopathy. Four different kinds of retinal conditions, viz., normal retina, moderate diabetic retinopathy, severe diabetic retinopathy (Latare \& Patil, 2015) and proliferative diabetic retinopathy (Walvekar \& Salunke, 2015). These are identified by image processing algorithms. Various aspects of image analysis tools were used in (Singh \& Tripathi, 2010 ) automated detection of various features and stages of diabetic retinopathy in retinal images. This includes the process of pre-processing, optic disc localization and segmentation, segmentation of retinal vasculature, localization of fovea and macula then detection of DR. Several papers and methods discussed about the detection of diabetic retinopathy. Here, we have discussed some of the state-of-the-art algorithms processes. Automatic detection of microaneurysms methods are described in (Sopharak, Uyyanonvara, Barman \& Williamson, 2011), (Aravind, PonniBala \& Vijayachitra, 2013), (lazar \& Hajdu, 2013), (Ruthra, 2014), (Manimala \& Gokulakrishna, 2014), (Giancardo et al., 2010), (Nemade \& Bhagat, 2015 ).

Akara Sopharak (Sopharak, Uyyanonvara, Barman \& Williamson, 2011) discussed about automatic micraneurysms detection from Non-dilated Diabetic Retinopathy Retinal Images. Non dilated pupils obtained from a KOWA-7 non-mydriatic retinal camera with a $45^{\circ}$ field of view. The image size is $752 \times 500$ pixels with 24 bits per pixel. MAs are difficult to detect because their contrast is very low and hardly visible and also difficult to distinguish from noise variations. This could not provide efficient accuracy and does not provide effective noise removal result due to no proper classification and detection methods.

Datta (Datta N., Dutta H. \& Mondal, 2013), proposed automated diabetic retinopathy screening system that shows the accurate results for classifying non-dilated retinal images to normal eye and diabetic eye.

Diabetic Retinopathy based on microaneurysms using SVM classifier. The SVM classifier (Aravind, PonniBala 
\& Vijayachitra, 2013) classifies the fundus images as normal, mild and severe based on the extracted features as input. In paper (lazar \& Hajdu, 2013) (lazar \& Hajdu, 2013) and (Ruthra, 2014) explained and analyzed about the directional cross section profiles. This method is an automated screening method for detecting diabetic retinopathy. By only considering the local maxima of the pre-processed image, the number of pixels to be processed is significantly reduced. Here, peak detection method is applied for each cross section profile and calculate a set of values that describe the size, height, and shape of the central peak. For screening Local Binary patterns (LBPs) are well known in retinal images (Morales, Engan, Naranjo \& Colomer, 2015).

Authors in (Mizutani et al., 2009) proposed a computerized method for the detection (CAD) of microneurysms on fundus images, which were obtained from the ROC database (Retinopathy Online Challenge) for training cases. Here 12 features extracted and candidate lesions were classified into microaneurysms or false positives. To overcome the sensitivity problem FPCM were proposed in paper (Pal N., Pal K., Keller \& Bezdek, 2005) and (Kumari, Sharma B. \& Gaur, 2012). We can avoid the scaling problem and make FPCM more efficient and useful for large data sets.

Meenakshi Sharma et al (Sharma M. \& Mukherjee, 2014) discussed overall methodologies for segmentation, feature extraction and feature selection in brain tumor. There are several algorithms and techniques proposed for image segmentation. They are Region growing, Thresholding, Binarization, Clustering methods, supervised learning algorithms and soft computing techniques (Fuzzy Logical Models, Neural Networks, Genetic Algorithm, Particle Swarm Optimization and Evolutionary Algorithms).

Several classification methods were used in DR that will discuss in (Priya \& Aruna, 2012) and (Janakiraman \& Gowri, 2014). Paper (Priya \& Aruna, 2012) proposed SVM classifier and PNN classifier for higher accurate classification. Support vector machine is analyzing data and predict an optimal way to classify the images. Probabilistic neural networks composed by many interconnected processing or neurons organized in successive layers. When compare SVM and PNN, SVM gives higher classification accuracy result than PNN.

\section{Proposed Work}

\subsection{Microaneurysms (MAs) Detection}

In experts point of view detection of MAs become difficult due to low contrast and noisy retinal images. In this paper image processing based Diabetes screening system is developed using MATLAB software for calculating the microaneurysms area on given retinal images. The overall System Architecture for MA detection is shown in figure 3. Our proposed system contains the following process of i) Preprocessing performed with the steps of the normalization, intensity conversion, denoising and contrast enhancement ii) Blood vessel segmentation iii) Fovea localization iv) Elimination of Fovea v) Feature extraction vi) Classification. These above mentioned processes are done with the help of an efficient image processing algorithms. Overall Architecture of our proposed system is depicted below:

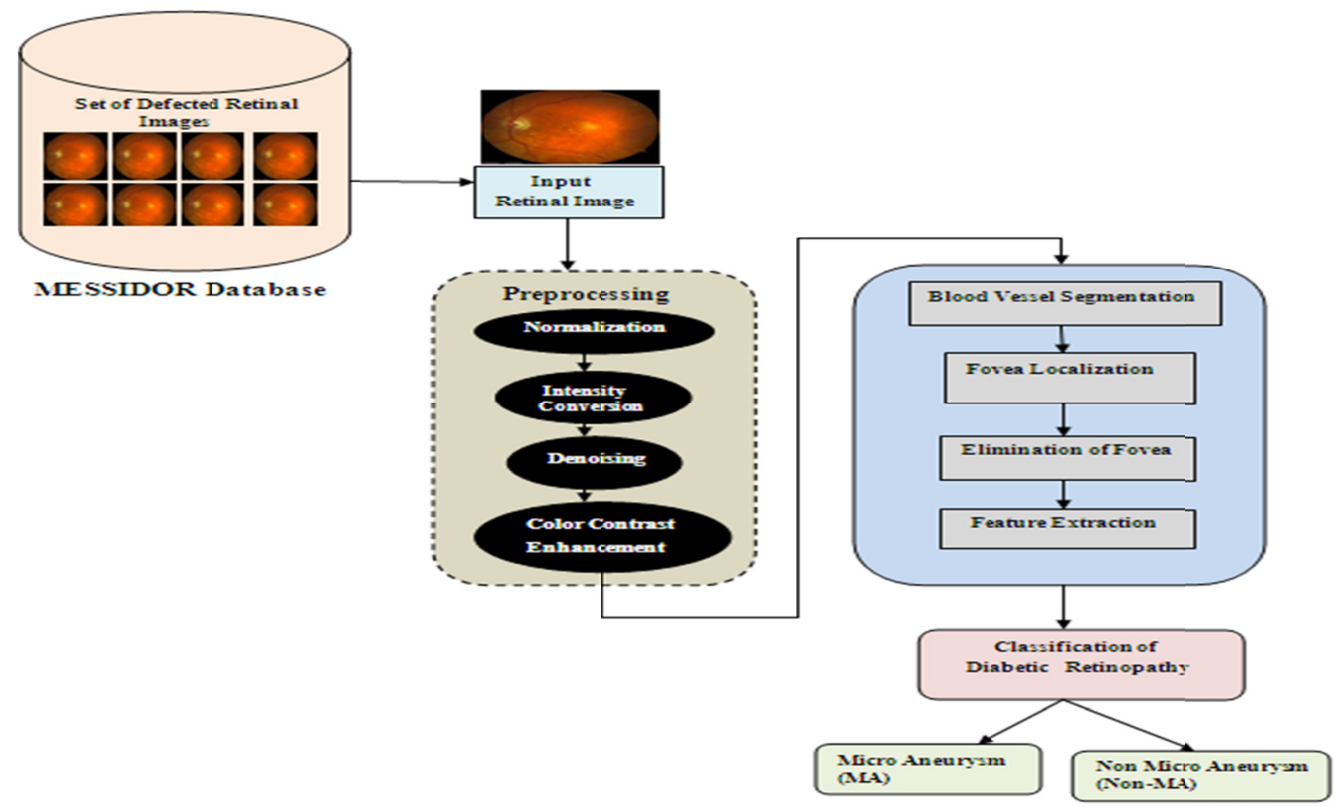

Figure 3. System architecture 


\section{A) Pre-Processing:}

Pre-Processing is an initial stage in most of the image processing techniques. Here, pre-processing consists of four steps:

(i) Normalization.

(ii) Intensity conversion.

(iii) Denoising using Neighboring wavelet coefficients.

(iv) Contrast Enhancement.

\section{(i) Normalization:}

In this step normalizes the non-uniformly distributed background by removing the bias field like region, which is acquired by convolve the original image.

\section{(ii) Intensity Conversion:}

In intensity conversion we first take the RGB retinal fundus image as input image which has RGB channels. Its green channel can emits best contrast among vessels and the background. Reason for not choosing other channels, they are too noisy. Green channel used to convert an intensity of an image.

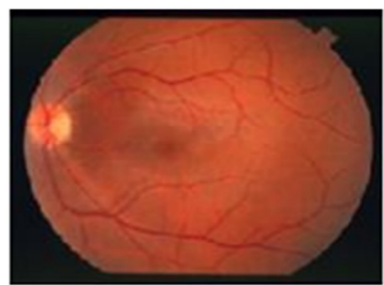

(a)

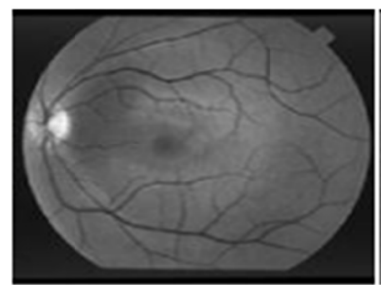

(b)

Figure 4. Intensity conversion (a) Input image, (b) Intensity image

(iii) Denoising:

Denoising is also an important stage in image processing, because all images taken from database which include noises. To remove noises we have to use neighboring wavelet coefficients. Selecting the threshold is an important for image denoising. There are several methods for image denoising in wavelet domains, such as Visu Shrink, Sure Shrink and Baye Shrink (kumar \& Saini, 2012). In this paper we introduce the Neigh Shrink method for modification of noisy image. The thresholding is undertaken by pixel by pixel basis when considering the influence of neighborhood wavelet coefficients.

Where $\mathrm{B}_{(\mathrm{i}, \mathrm{j})}$ is the Shrinkage factor it can be defined as,

$$
\mathbf{B}_{(\mathrm{i}, \mathrm{j})}=\left(\lambda^{2} / S_{(i, j)}^{2}\right)
$$

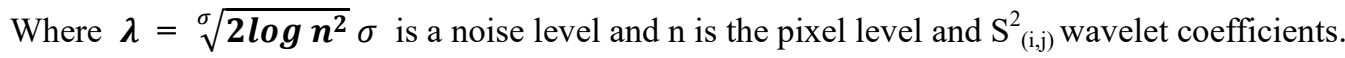

\section{(iv) Contrast Enhancement:}

Contrast enhancement is improves the interpretability or perception in images. There are four techniques used for contrast enhancement i.e. Linear Contrast Stretching, Histogram Equalization, Contrast Limited Adaptive Histogram Equalization (CLAHE), Brightness Preserving Histogram Equalization. The best technique among the above discussed technique is Brightness Preserving Histogram Equalization (BPHE). It produces good result while preserving mean brightness in the retinal images. BPHE is used to overcome the problem of histogram equalization. It computes the mean value of the particular image and decomposes retinal image into two sub images based on the mean value. Finally equalized sub images are bounded by each other around the mean, which has an effect of preserving mean brightness. 


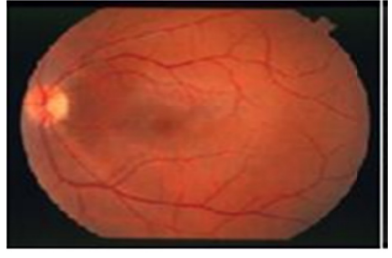

(a)

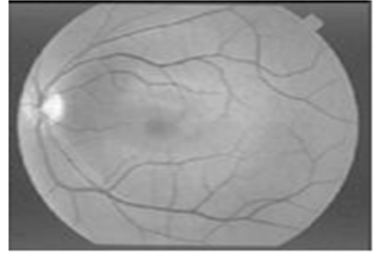

(b)

Figure 5. Contrast Enhancement, (a) Input image, (b) Contrast enhanced image

\section{B) Blood Vessel Segmentation:}

Blood vessel segmentation is used to get only the blood vessel with black background. This vessel segmentation process is to be done with the database of MEISSDOR. Fuzzy Possibilistic C-Means (FPCM) algorithm should ensure the blood vessel segmentation.

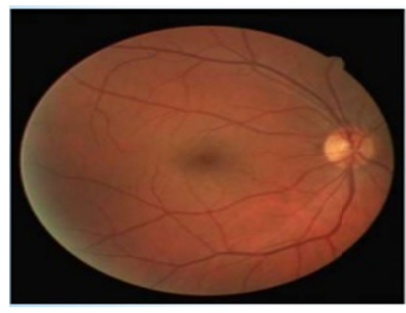

(a)

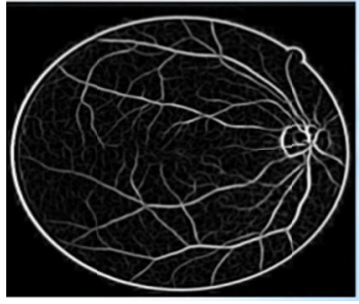

(b)

Figure 6. Blood Vessel Segmentation, (a) Input image, (b) Blood vessel segmented image

A fuzzy similarity-based self-constructing feature clustering technique is used in incremental feature clustering method. It reduces the amount of feature for the text classification approach. An improved FPCM algorithm is split a specified set of data or objects into a cluster for the purpose of a clustering analysis, which signifies subsets or a group.

FPCM segmentation process should be mention below:

Step 1: Objective function of optimization problem can be stated below,

$$
\min _{(U, T, V)}\left\{J_{m, n}^{(U, T, V)}=\sum_{i=1}^{c} \sum_{k=1}^{n}\left(u_{i k}^{m}+t_{i k}^{n}\right) D_{i k}^{2}\right\}
$$

Where $u_{i k}$ is a function of $X_{k}$ and all centroids c. $t_{i, k}$ is a function of $X_{k}$ and $V_{i}$ alone. In above mentioned notations are important to classify a data point and cluster centroid. Since cluster centroid $\mathrm{c}$ is closest to the data point $\mathrm{n}$.

Step 2: Calculate the membership function by using,

$$
\mathbf{u}_{\mathrm{ik}}=\left[\sum_{j=1}^{c}\left(\frac{D_{i k}}{D_{j k}}\right)^{\frac{2}{m-1}}\right]^{-1}, \forall i, k
$$

Step 3: Calculate typicality function by using,

$$
\mathbf{t}_{\mathrm{ik}}=\left[\sum_{j=1}^{n}\left(\frac{D_{i k}}{D_{i j}}\right)^{\frac{2}{n-1}}\right]^{-1}, \forall i, k
$$

Constraints for membership function and typicality are $\sum_{i=1}^{c} \boldsymbol{u}_{i k}=\mathbf{1}, \forall k, \sum_{i=1}^{n} \boldsymbol{t}_{i k}=\mathbf{1}, \forall \boldsymbol{i}$ respectively. Because of this constraint, typicality of a data point to a cluster will be normalized with respect to the distance of all $\mathrm{n}$ data points from that cluster. 
Step 4: Compute the cluster centroids by using,

$$
\mathbf{V}_{\mathrm{i}}=\left(\sum_{k=1}^{n}\left(u_{i k}^{m}+t_{i k}^{m}\right) X_{k} / \sum_{k=1}^{n}\left(u_{i k}^{m}+t_{i k}^{m}\right)\right), \forall i
$$

We have to overcome the scaling and sensitivity problem by giving higher values to the typicality and membership alternatively it will reduce the outliers. Scaling optimization problem also reduced and it is applicable for large datasets (Pal N., Pal K., Keller \& Bezdek, 2005 ) and (Kumari, Sharma B. \& Gaur, 2012). According to the above mentioned process retinal blood vessels are segmented properly.

\section{C) Fovea Localization:}

Fovea is the most essential part of retina for human vision since it detected from blood vessels. The size of fovea zone in fundus image is very small and it determines whether it may lead to various diseases, which may turn out to blindness. Geometrically fovea is at a distance of 2.5 times the diameter of the Optic Disk (OD) from its center. The center of the fovea region is localized and marked red in Figure (7). The algorithm is as follows.

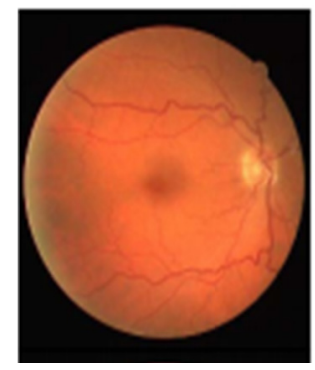

(a)

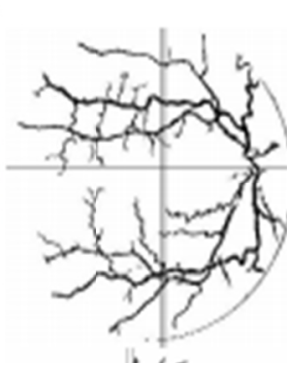

(b)

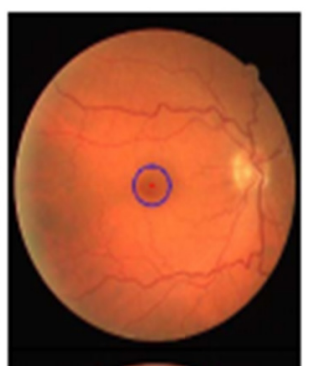

(c)

Figure 7. Fovea Localization, (a) Input image, (b) Blood vessels, horizontal line and Vertical strip, $\quad$ (c) Fovea Localized

Algorithm for Fovea Localization:

Input: Fundus image, an image (contains only blood vessels), approximate center (C) and diameter (D) of the optic disk.

Output: Fovea region is localized (Vipin, Jayanthi \& Kunjummen, 2013).

Step1: Locate a point $\mathrm{P}_{\mathrm{t}}$ horizontally at a distance $2.5 \times \mathrm{D}$ from $\mathrm{c}$ towards the centroid.

Step2: Consider a vertical strip of width k pixels around $\mathrm{P}_{\mathrm{t}}$ perpendicular to $\mathrm{CP}_{\mathrm{t}}$.

Step3: Apply an $n \times n$ sliding window along the strip and form the chain of numbers denoting the black pixels in the window.

Step4: Find the maximum run length of zeros, $\mathrm{N}$ in the number chain.

Step5: Let $S_{t}$ and $E_{d}$ are the start and end position corresponding to $\mathrm{N}$ and $\mathrm{D}$ is the mid position of $\mathrm{S}$ and $\mathrm{E}_{\mathrm{d}}$.

Step6: Detect fovea regions as the small area around the center of macula.

\section{D) Elimination of Fovea:}

Fovea elimination is a main process for finding microaneurysms in the retinal images. Because, usually fovea looks like hemorrhage in color but different in regions. For that purpose we have to eliminate the fovea. First, fovea center has to be identified using morphological dilation operation. Dilation operation is to perform with binary image and represented by centre point which is called fovea centre. Dilation structuring element has radius of 25 pixels, fovea has circular characteristics and also has similar size to this value. This fovea can be eliminated from the masking of dilation process with the fovea region.

\section{E) Feature Extraction:}

In feature extraction texture is the main feature of an image. There are several techniques proposed for measuring texture in various research fields such as co-occurrence matrix, fractals, gobor filters and wavelet transform (Sharma M. \& Mukherjee, 2014). After fovea localization, the feature extraction is computed using GLCM and LBP. Feature extractions in the basis of color and texture orientation feature are extracted using 


\section{GLCM and LBP.}

\section{(i) Local Binary Pattern (LBP):}

Local Binary Pattern is a non parametric grey-scale texture operator, which is efficiently summarizes the local structure of images. The local binary pattern operator is a powerful tool in texture description. In fundus images LBP is extract texture features from the blood vessels that are shown in figure 8. LBP is very sensitive in noise because the operator thresholds exactly at the value of central pixel. An LBP is called uniform pattern since the binary pattern contains at most two bit wise transitions from 0 to 1 or vice versa when the bit pattern is considered circular. LBP that uses values of each pixel and its neighbor's pixels $p$ and radius $r$.

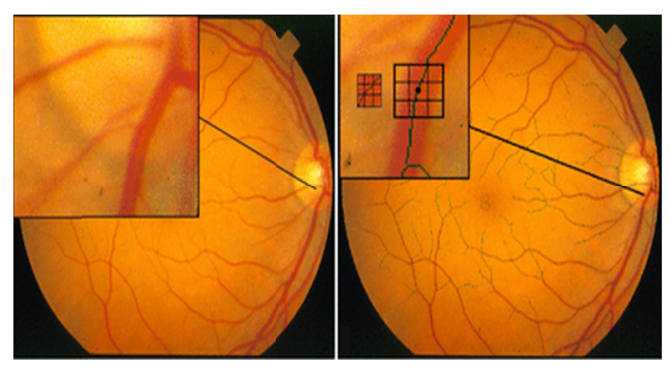

Figure 8. Feature extracted in blood vessels fundus image

$$
\mathrm{LBP}_{\mathrm{P}, \mathrm{R}}=\sum_{p=0}^{P-1} S\left(G_{p}-G_{c}\right) 2^{p}, \mathrm{~S}(\mathrm{X})=\left\{\begin{array}{l}
1 \text { if } X \geq 0 \\
0 \text { if } X<0
\end{array}\right.
$$

Where $G_{p}$ and $G_{c}$ are the grey values of the neighborhood and central pixel respectively. p represents the number of samples on the symmetric circular neighborhood of radius r. $2^{\mathrm{P}}$ is the binary patterns that generated for each neighborhood.

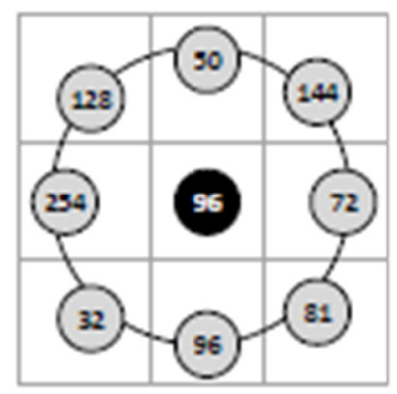

(a)

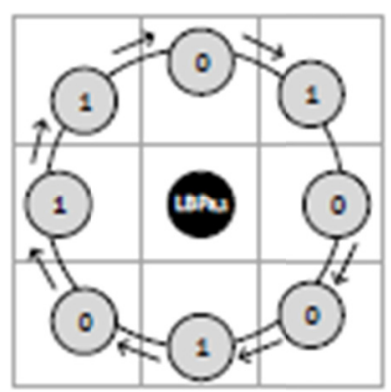

(b)

Figure 9. LBP computation: (a) Grey values of a circular neighborhood of radius 1 and 8 samples, (b)

Thresholding between the grey value of the neighborhood and the central pixel

LBP Computation: Binary code word $=01001011$, LBP value $=1 \times 2^{1}+1 \times 2^{4}+1 \times 2^{6} \times 2^{7}=210$.

(ii) Grey Level Co-occurrence Matrix (GLCM):

GLCM also focuses on texture information. It is a matrix where the number of rows and columns equal to the number of grey levels. The matrix element is relative frequency in which two pixel are separated by pixel distance one with intensity $i$ and another one with intensity $j$. A co-occurrence matrix over an $n \times m$ in image $i$. Where $\mathrm{n}$ is the neighbor point value and $\mathrm{m}$ is the reference point value. GLCM captures numerical feature values according to their texture features. GLCM extract 10 texture features such as Contrast, Correlation, Dissimilarity, Entropy, Homogeneity, Maximum probability, Angular Second Moment and Mean which are calculated using feature function. 
Table 1. GLCM matrix

\begin{tabular}{llllll}
\hline NPV/RPV & $\mathbf{0}$ & $\mathbf{1}$ & $\mathbf{2}$ & $\mathbf{3}$ & $\mathbf{4}$ \\
\hline $\mathbf{0}$ & $(0,0)$ & $(0,1)$ & $(0,2)$ & $(0,3)$ & $(0,4)$ \\
$\mathbf{1}$ & $(1,0)$ & $(1,1)$ & $(1,2)$ & $(1,3)$ & $(1,4)$ \\
$\mathbf{2}$ & $(2,0)$ & $(2,1)$ & $(2,2)$ & $(2,3)$ & $(2,4)$ \\
$\mathbf{3}$ & $(3,0)$ & $(3,1)$ & $(3,2)$ & $(3,3)$ & $(3,4)$ \\
$\mathbf{4}$ & $(4,0)$ & $(4,1)$ & $(4,2)$ & $(4,3)$ & $(4,4)$ \\
\hline
\end{tabular}

\section{(I) Maximum Probability (MP):}

Probability between NPV and RPV and its maximum value is taken for feature value.

$$
\mathbf{F}(\mathbf{x}, \mathbf{y})=\max _{i, j} p(x, y)
$$

Where $\mathrm{x}, \mathrm{y}$ denotes the NPV and RPV respectively and $\mathrm{p}(\mathrm{x}, \mathrm{y})$ denotes the probability of maximum pixel value(NPV and RPV)

\section{(II) Contrast (Cn):}

$$
\mathbf{F}(\mathbf{x}, \mathbf{y})=\sum_{i} \sum_{j}\left((\boldsymbol{i}-\boldsymbol{j})^{2} \boldsymbol{p}(\boldsymbol{x}, \boldsymbol{y})\right.
$$

(III) Entropy (Ep):

$$
\mathbf{F}(\mathbf{x}, \mathbf{y})=\sum_{i} \sum_{j}\left(\frac{p(x, y)}{\log p(x, y)}\right)
$$

IV) Angular Second Moment (Asm):

$$
\mathrm{F}(\mathbf{x}, \mathbf{y})=\sum_{i} \sum_{j}(x, y) 2
$$

v) Homogeneity (Hm):

VI) Dissimilarity (Ds):

$$
\mathbf{F}(\mathbf{x}, \mathbf{y})=\sum_{i} \sum_{j}\left(\frac{p(x, y)}{1+|i-j|}\right)
$$

VII) Mean (Mn):

$$
\mathbf{F}(\mathbf{x}, \mathbf{y})=\sum_{i} \sum_{j}|\boldsymbol{i}-\boldsymbol{j}| \boldsymbol{p}(\boldsymbol{x}, \boldsymbol{y})
$$

$$
\mathbf{F}(\mathbf{x}, \mathbf{y})=\frac{\sum_{i} \sum_{j} p(x, y)}{n \times m}
$$

Where $\mathrm{n}$ is the number of rows and $\mathrm{m}$ is the number of columns.

\section{VIII) Correlation (Cr):}

$$
\mathbf{F}(\mathbf{x}, \mathbf{y})=\sum_{i} \sum_{j} \frac{\left(i-\mu_{x}\right)\left(j-\mu_{y}\right) p(x, y)}{\sigma_{x} \sigma_{y}}
$$

To create GLCM: GLCM = graycomatrix (image I, 'offset value [])

\section{F) Classification:}

Classification is a final step of our DR disease identification process. After extracting the features we have to classify diseases into Microaneurysms and Non-Microaneurysms. For classification purpose, features as an input that is fed to a classifier based on neural network and fuzzy logical models. Fuzzy logics and neural networks are natural complementary tools in building for classification. Neural networks and fuzzy logic are two approaches that are widely used to solve classification problem. While networks are low-level computational structures that perform well when dealing with raw data, fuzzy logic deals with reasoning on a higher level. A fuzzy logical system is a non-linear mapping of features into a scalar output. The fuzzy model is used for giving more accurate results when adding more number of features into fuzzy models. The main advantage of neural networks is their learning capabilities and their ease of implementation. When combined neural networks and fuzzy logic it exploits more number of advantages.

Feature sets such as Mp, Em, Ep, Asm, Hm, Ds, Mn and Cr are extracted from the input images (fundus image) using GLCM. The fundus images are classified using the Neuro-Fuzzy classifier. Extracted features are given as the input to the Neuro-Fuzzy Classifier which is classified by all the given fundus images into 2 classes 
i.e.Microaneurysms and Non-Microaneurysms. Neuro-Fuzzy classifier is a fuzzy based system that is trained by a learning algorithm derived from Neural Networks. Learning algorithm only performs on the local information and provides local modifications in the fuzzy system. In general, a neuro-fuzzy system generates very powerful solutions instead of using the system components individually. Input feature values are fuzzified using membership functions (MF) that are facilitated by the membership of each feature to different classes. Unknown and inter- related information are extracted from the features to the classes through the MF, which leads to get more accuracy of the classification using Neuro-Fuzzy classifier. The membership matrix is computed with 8 rows and 2 columns, in which the number of rows is equal to the number of features and the number of columns is equal to the number of classes. Membership matrix MF produced degree of different features (dF) to different classes(C) (Bhanumurthy \& Koteswararaa, 2014).

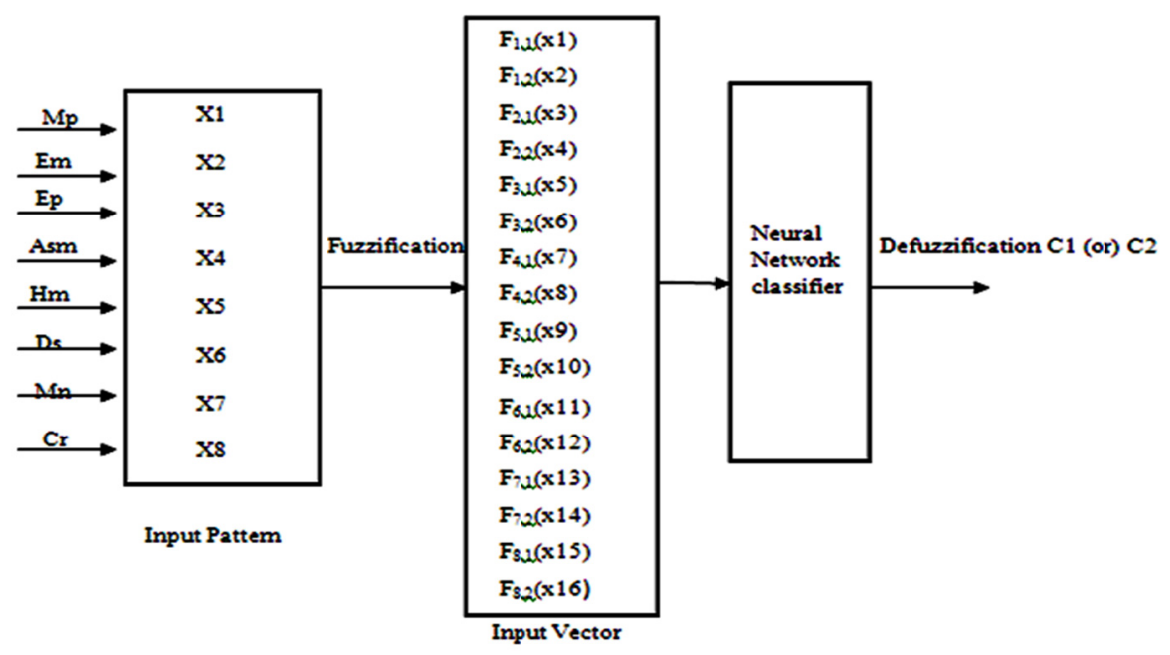

Figure 10. Neuro-Fuzzy System

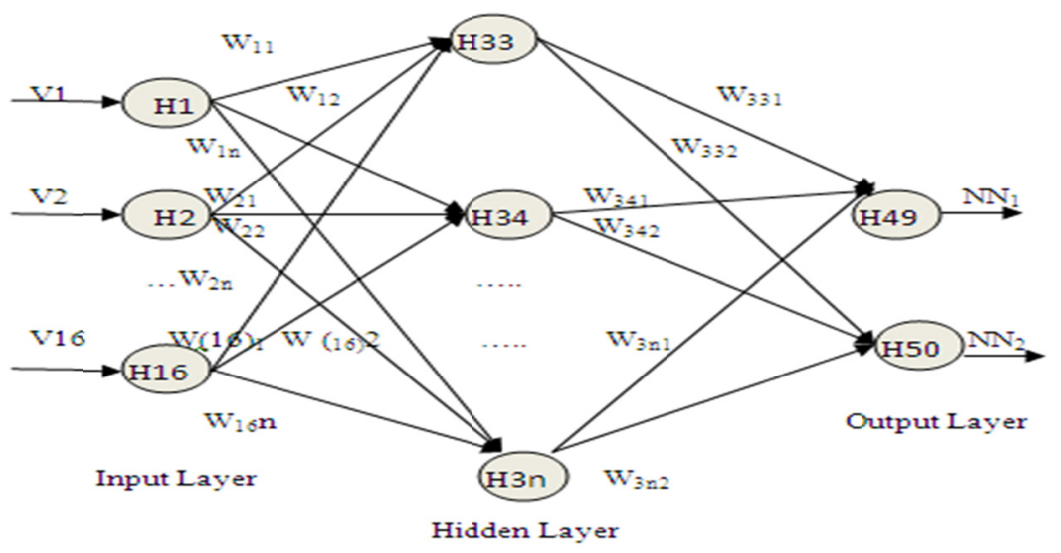

Figure 11. Neural Network Classifier

All rows and columns in the membership matrix are cascaded and converted into a vector (v1, v2 .. v16). These vectors are given as the input to the Neural Network (NN). In this neural network Multi-Layer Perception (MLP) classifier is used. MLP has three layers such as input layer (vectors), hidden layer (weights for input vectors) and output layer (Neural networks for class 1 and class 2). The total number of input nodes of the $\mathrm{NN}$ is equal to the product of 8 features and 2 classes are 16, which is the number of input nodes of the NN. The total number of output nodes from the NN is same as that of the number of classes, and here two output nodes are generated from the NN. The total number of hidden nodes is equal to the square root of the product, of the number of input nodes and output nodes. Defuzzification process is carried out on the output nodes of NN, by performing a max operation. The output is a single value, $\mathrm{c} 1$ or $\mathrm{c} 2$ for a given fundus image. From this value, we can able to 
classify whether the given input fundus image is Microaneurysms or Non-Microaneurysms (Bhanumurthy \& Koteswararaa, 2014).

\section{Experimental Analysis}

Our proposed Microaneurysms detection and classification of retinal disease should be processed by segmentation and classification steps. Initially pre-processing fundus image by reduces the noise level and also performed with contrast enhancement. Blood vessel segmentation done with the help of Fuzzy Possibilistic C-Means should contain segmentation information about every retinal image. In classification of diabetic retinopathy we have to use Neuro-Fuzzy classifier which increases sensitivity and accuracy. Comparison shall be made for classification. For classification we have to compare previous classifiers with our Neuro-Fuzzy.

\subsection{Database}

MESSIDOR database (Methods for Evaluating Segmentation and Indexing techniques for Dedicated to Retinal Ophthalmology) is used in our proposed system. Generally, MESSIDOR database consists of two sets of data such as training set and evaluation set. Here, we evaluate this database for identifying Microneurysms and Non-Microneurysms. MESSIDOR database contains 1200 retinal images, which is the largest database publicly available on the internet. The images will be saved as uncompressed TIFF format with a $1440 * 960$ pixel resolution that is about $4 \mathrm{MB}$ per image. These images acquired by 3 ophthalmologic departments using a color video 3CCD camera on a Topcon TRC NW6 non-mydriatic retinograph with a $45^{\circ}$ Field of View (FOV). The images captured using 8 bit per color on plane at the pixel ranges are $1440 * 960,2240 * 1488,2304 * 1536.1200$ images are divided into two sub images i.e. 800 images acquired with pupil dilation (one drop of Tropicamide at $0.5 \%)$ and 400 images are without dilation.

\section{(i) Training Set:}

This dataset is used for testing and improving the available algorithms as well as for validating the methods used to evaluate the algorithms. This database contains 200 images.

For each image, it indicated at least:

- Stage of Diabetic Retinopathy.

- The number of and/or the surface of the micro aneurysms and Non-Microaneurysms.

\section{(ii) Evaluation Set:}

This dataset contains thousand images since its purpose is evaluating the proposed algorithms.

Usually, Diabetic Retinopathy stages are classified as mild, moderate, severe and PDR. But in our proposed process we have to categorize diseases like Microaneurysms and Non-Microaneurysms. Our dataset is processed and improve classification accuracy.

\subsection{Performance Metrics}

Performance of the test classifier can be measured in the form of sensitivity (or) Recall, specificity and Accuracy (Correct classification and Detection rate). True positive (TP), True Negative (TN), False Positive (FP) and False Negative (FN) are the test outcomes. In general True positive is correctly identified, False Positive is incorrectly identified, True negative is correctly rejected and finally false negative is incorrectly rejected. Performance metrics such as sensitivity, specificity and accuracy are in follows:

\section{(i) Sensitivity:}

Sensitivity can be measured by the proportion of positives, disease affected peoples can be correctly identified.

This can be computed as follows:

$$
\text { Sensitivity }(\%)=\frac{T P}{T P+F N} * 100 \%
$$

\section{(ii) Specificity:}

Specificity can be measured by the proportion of negatives, peoples does not could affected are correctly identified. This can be computed as follows:

$$
\text { Specificity }(\%)=\frac{T N}{T N+F P} * 100 \%
$$




\section{(iii) Accuracy (Correct classification and detection rate):}

Accuracy can be measure the overall performance of our proposed sot computing approaches. This can be computed as follows:

$$
\text { Accuracy }(\%)=\frac{T P+T N}{T P+T N+F P+F N} * 100 \%
$$

\subsection{Comparative Analysis}

In our experiments comparative analysis shall be done with some soft computing approaches which can be used in our proposed work with state-of-the art techniques. Here, we have to compare the detection stage algorithms (SVM and Neuro-Fuzzy). Before comparing these methods we produce our results on every stage. Fig. displayed the Preprocessing results of our proposed system.

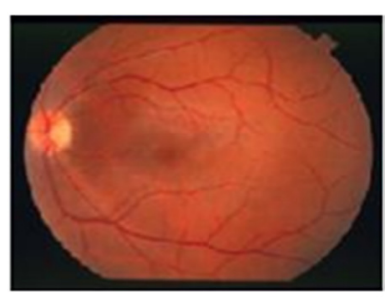

(a)

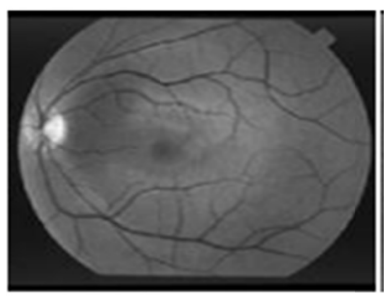

(b)

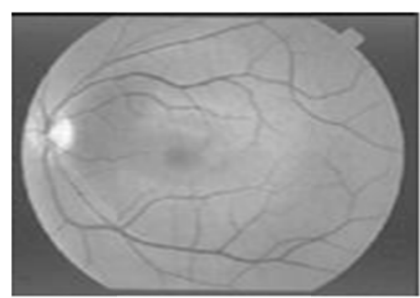

(c)

Figure 12. Pre-Processing Results a) Original image b) Intensity Conversion c) Contrast Enhancement

Blood vessel segmentation could be done with the help of FPCM. This FPCM overcomes sensitivity problem and improves scalability performance and Fovea localization can be done with the help of sliding window approach. In which image is divided into horizontal line and vertical strip. Results of Blood vessel segmentation and fovea localization can be depicted in figure 13 .

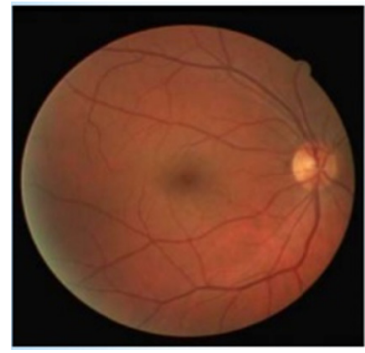

(a)

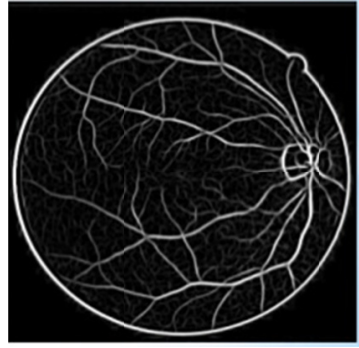

(b)

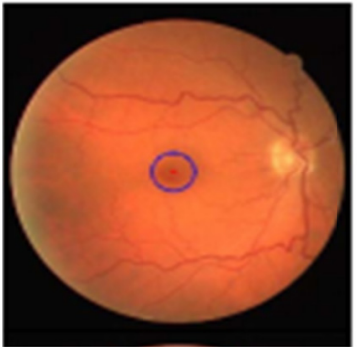

(c)

Figure 13. (a) Input image, (b) Blood vessel segmentation result, (c) Fovea localization result

In feature extraction, textural features such as contrast, homogeneity, correlation, mean, maximum probability, dissimilarity, angular second moment, entropy are calculated using GLCM and LBP. The features of 25 normal images and 25 DR images are given for training sets to the Neuro-Fuzzy classifier. For evaluating sets, the feature extracted from 75 normal images and 75 DR images are fed into the Neuro-Fuzzy Classifier. Classification comparison can be made with SVM (Support Vector Machine) and PNN (Probabilistic Neural Networks) classifier (Priya \& Aruna, 2012). Table 2 shows the accuracy results for proposed Neuro-Fuzzy classifier. Classification accuracy for normal images and diabetic retinopathy images are $99 \%$ and $99 \%$ respectively. Since the average accuracy for classification is $99 \%$. 
Table2. Classification results using Neuro- Fuzzy classifier

\begin{tabular}{cccccc} 
Classifiers & Classes & $\begin{array}{c}\text { No. of Training } \\
\text { Images }\end{array}$ & $\begin{array}{c}\text { No. of Testing } \\
\text { Images }\end{array}$ & $\begin{array}{c}\text { No. of correctly } \\
\text { Classified Images }\end{array}$ & $\begin{array}{c}\text { Classification } \\
\text { Accuracy }(\%)\end{array}$ \\
& Normal & 25 & 75 & 74 & 99 \\
Neuro-Fuzzy & DR & 25 & 75 & 74 & 99 \\
& & & Average Accuracy $=\mathbf{9 9 \%}$ & \\
\hline
\end{tabular}

Performance metrics compares with PNN, SVM and our proposed system (Priya \& Aruna, 2012). Table 3 shows our proposed system proves better results in sensitivity, specificity and Accuracy.

Table 3. Comparison of Sensitivity, specificity and Accuracy

\begin{tabular}{cccc} 
Methods & Sensitivity (\%) & Specificity (\%) & Accuracy (\%) \\
\hline PNN & 90 & 88 & 89.6 \\
SVM & 98 & 96 & 97.6 \\
Neuro-Fuzzy & 99 & 96 & 99 \\
\hline
\end{tabular}

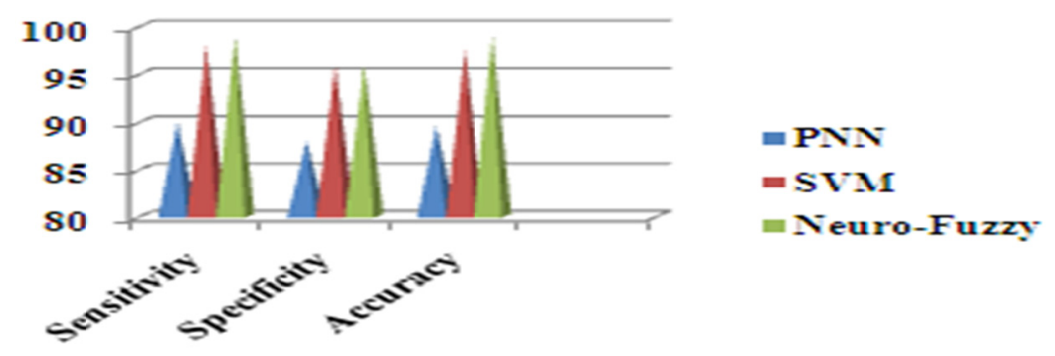

Figure 14. Comparison of Classification Process

Results of DR in Microaneurysms classified and displayed in figure 15.

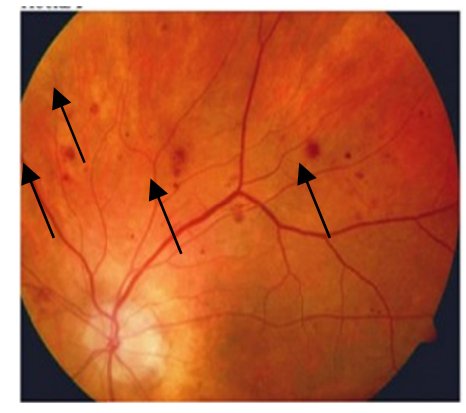

(a)

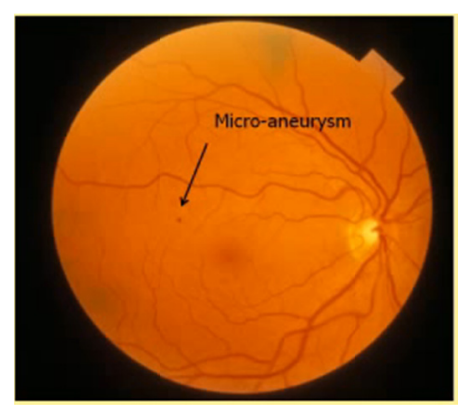

(b)

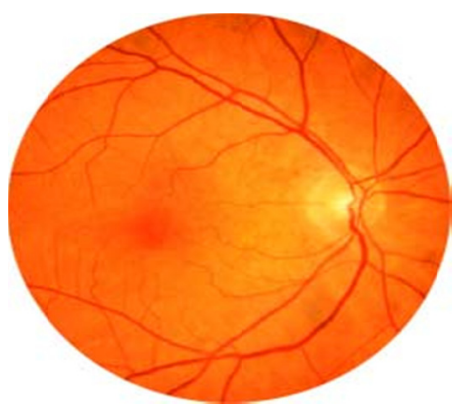

(c)

Figure 15. (a) \& (b) Microaneurysms (c) Normal Retinal Image

\section{Conclusion}

Our Proposed framework effectively discusses Microaneurysms disease detection and classification processes. Here, we improve accuracy of classification. To improve sensitivity and specificity our proposed framework encourages the methods such as blood vessel segmentation using Fuzzy Possibilistic C-Means, fovea localization (Sliding window), fovea elimination, feature extraction (LBP \& GLCM) and according to that features we can classify the disease easily this aws done in classification process. As an achievement of this work, the DR has been classified into two categories Microaneurysms and Non-Microanerysms using Neuro-Fuzzy classifiers. These two procedures utilized for the classification have high accuracy. Our proposed work achieves higher sensitivity of 
$99 \%$, specificity of $99 \%$ and accuracy is $99 \%$. In future, we will decide to separate the diseases with some different properties and use different database for processing.

\section{References}

Aravind, C., PonniBala, M., \& Vijayachitra, S. (2013). Automatic detection of microaneurysms and classification of diabetic retinopathy images using SVM technique. International Journal of Computer Applications, 18-22.

Bhanumurtht, M. Y., \& Koteswararoa, A. (2014). A Novel Method of Brain Tumor Segmentation Using Extensive Features Set. AIVP, Advances in Image and Video Processing, 2(5), 11-26. http//dx.doi.org/10.14738/aivp.25.445

Datta, N. S., Dutta, H. S., De, M., \& Mondal, S. (2013). An effective approach: Image quality enhancement for microaneurysms detection of non-dilated retinal fundus image. CIMTA, Procedia Technology, 10, 731-737. http://dx.doi.org/10.1016/j.protcy.2013.12.416

Ganesh, S., \& Basha, A. M. (2015). Automated detection of diabetic retinopathy using retinal optical images. International Journal of Science, Technology \& Management, 4(2), 136-144.

Giancardo, L., Meriaudeau, F., Karnowski, T., Tobin, K., Li, Y., \& Chaum, E. (2010). Microaneurysms detection with the Radon Cliff operator in retinal fundus images. Proc. of SPIE, Medical Imaging, 7623, 1-8 . http://dx.doi.org/10.1117/12.844442

Gowri, J. (2014). Robust color image segmentation using efficient soft-computing techniques: A survey. American International Journal of Research in Science, Technology, Engineering \& Mathematics, 5(2), 135-139.

Kumar, R., \& Saini, B. S. (2012). Improved image denoising technique using neighboring wavelet coefficients of optimal wavelet with adaptive thresholding. International Journal of Computer Theory and Engineering, 4(3), 395-400.

Kumari, N., Sharma, B., \& Gaur, D. (2012). Implementation of Possibilistic Fuzzy C-Means Clustering algorithm in Matlab. International Journal of Scientific \& Engineering Research, 3, 1-9.

Latare, K. R., \& Patil, W. V. (2015). A Novel approach for the detection \& classification of diabetic retinopathy. International Journal on Recent and Innovation trends in Computing and Communication, 3, 958-961.

Lazar, I., \& Hajdu, A. (2013). Retinal microaneurysm detection through local rotating cross-section profile analysis. Transactions on Medical Imaging, 32(2), 400-407.

Maheswari, M. S., \& Punnolil, A. (2014). A Novel approach for retinal lesion detection diabetic retinopathy images. International Journal of Innovative Research in Science, Engineering and Technology, 3, 1109-1114.

Mamatha, B. V., Srilatha, L. R., Devaraj, D., \& Kumar, P. (2015). A survey on different classifiers for medical diagnosis and grading: Application to diabetic retinopathy. International Journal of Healthcare Sciences IJHS, 2(2), 210-216.

Manimala, K., \& Gokulakrishna, K. (2014). Detection of microaneurysms in color fundus images. International Journal of Emerging Technology and advanced Engineering, 4, 30-37.

MESSIDOR Database, http://messidor.crihan.fr/download.php

Mizutani, A., Muramatsu, C., Hatanaka, Y., Suemori, S., Hara, T., \& Fujita, H. (2009). Automated microaneurysm detection method nased on double-ring filter in retinal fundus images. Proceedings of SPIE, Medical Imaging, 7260, 7261N/1-72601N/8.

Morales, S., Engan, K., Naranjo, V., \& Colomer, A. (2015). Retinal disease screening through local binary patterns. IEEE Journal of Biomedical and Health Informatics, 0, 1-8. http://dx.doi.org/10.1109/JBHI.2015.2490798

Nemade, K., \& Bhagat, K. S. (2015). Microaneurysms Detection from Retinal Image and Diabetic Retinopathy Grading. International Journal of Emerging Trends \& Technology in Computer Science, 4, 74-77.

Oliver, F., Rajendra, A. U., E. Y. K., Ng, Kwan-Hoong, Ng, \& Jasjit, S. S. (2012). Algorithms for the Automated Detection of Diabetic Retinopathy Using Digital Fundus Images: A Review. Journal of Medical Systems, 36, 145-157. http://dx.doi.org/10.1007/s10916-010-9454-7

Pal, N. R., Pal, K., Keller, M. K., \& Bezdek, J. C. (2005). A Possibilistic Fuzzy C-Means clustering algorithm. 
IEEE Transaction on Fuzzy Systems, 13(4), 517-530.

Paranjpe, M. J. \& Kakatkar, M. N. (2014). Review of methods for diabetic retinopathy detection and severity classification. International Journal of Research in Engineering and Technology, 3, 619-624.

Priya, R. \& Aruna, P. (2012). SVM and Neural Network Based Diagnosis of Diabetic Retinopathy. International Journal of Computer Applications, 41(1), 6-12.

Rajab, A. (2015). Detection of diabetic retinopathy in fundus image. International Journal of Science and Application, 1(1), 26-30.

Ruthra, K. (2014). Retinal microaneurysm exclusion on optic disc and detection using cross section profile analysis. International Journal of Innovative Research in Computer and Communication Engineering, 2, 2069-2074.

Sharma, M., \& Mukherjee, S. (2014). Fuzzy C-Means, ANFIS and Genetic Algorithm for segmenting astrocytoma - A type of brain tumor. International Journal of Artificial Intelligence, 3(1), 16-23.

Singh, N., \& Tripathi, R. C. (2010). Automated early detection of diabetic retinopathy using image analysis techniques. International Journal of Computer Applications, 8(2), 18-23.

Sirajudeen, A., \& Ezhilarasi, M. (2015). Multi-scale LBP and SVM classifier to identify diabetic retinopathy in lesions. Current Medical Imaging Reviews, 11, 49-55.

Sopharak, A., Uyyanonvara, B., Barman, S., \& Williamson, T. (2011). Automatic microaneurysm detection from non-dilated diabetic retinopathy retinal images. Proceedings of the World Congress on Engineering, 2.

Verma, K., Deep, P., \& Ramakrishnan, A. G. (2011). Detection and classification of diabetic retinopathy using retinal images. India Conference, 1-6. http://dx.doi.org/10.1109/indcon.2011.6139346

Vipin, C. V., Jayanthi, V. S., \& Kunjummen, J. V. (2013). Iterative Kernel PCA based Classification of Retinal Images for Diabetic Macular Edema. International Journal of Computer Applications, 67(8), 2013, 22-26.

Walvekar, M., \& Salunke, G. (2015). Detection of diabetic retinopathy with feature extraction using image processing. International Journal of Emerging Technology and Advanced Engineering, 5, 133-137.

Yun, W. L., Acharya, U. R., Venkatesh, Y. V., Chee, C., Min, L. C., \& Ng, E. Y. (2008). Identification of different stages of diabetic retinopathy using retinal optical images. Information Sciences, 178, 106-121. http://dx.doi.org/10.1016/j.ins.2007.07.020

\section{Copyrights}

Copyright for this article is retained by the author(s), with first publication rights granted to the journal.

This is an open-access article distributed under the terms and conditions of the Creative Commons Attribution license (http://creativecommons.org/licenses/by/4.0/). 